\title{
Acute Promyelocytic Leukaemia: A Large Case Series from Southern India Based on Clinical, Pathological, Immunophenotypic, Cytogenetic and Molecular Studies
}

T SRAVANI ${ }^{1}$, PAMU PRAMOD KUMAR ${ }^{2}$, ASWANI TANDON ${ }^{3}$, MEGHA S UPPIN ${ }^{4}$, SHANTIVEER G UPPIN ${ }^{5}$,

AMVR NARENDRA ${ }^{6}$, GUNDETI SADASHIVUDU7 ${ }^{7}$ T ROSHNI PAUL ${ }^{8}$

\section{ABSTRACT}

Introduction: Acute Promyelocytic Leukaemia (APL/APML) is an uncommon haematologic malignancy associated with translocation $t(15 ; 17)$ and presence of abnormal promyelocytes in the peripheral blood and bone marrow.

Aim: To know the clinico-pathological profile of all cases diagnosed as APL and correlate the morphology with the cytogenetic and molecular studies.

Materials and Methods: It was a retrospective cum prospective analytical study done over a period of five years. Initially all cases were diagnosed on basis of morphology and later confirmed by immuno-phenotyping and molecular characterisation. Results were analysed based on morphological distribution of the cases, Immuno-chemistry and cyto-genetics.

Results: During the study period, 125 cases were diagnosed as APL. The patient's ages ranged from 3 years -60 years (median age 35 years) with M: F ratio of $1.4: 1$. Common clinical presentations were fever, bleeding manifestations and anaemia. Total leucocytes count varied with cases; 56 cases presented with leucopenia, 44 cases with leucocytosis and 25 cases with normal leucocytes counts. Most of the cases showed marked thrombocytopenia. The cases were classified as hypergranular (80 cases), microgranular (39 cases) and APL Not Otherwise Specified (NOS) with atypical features (6 cases). Sudan Black B (SBB) was intensely positive in $100 \%$ of cases. Promyelocytic Leukaemia/Retinoic Acid Receptor Alpha (PML-RARA) by Flourescence In-Situ Hybridization (FISH) and Polymerase Chain Reaction (PCR) testing was positive in $95.5 \%$ of available cases. Other cases were diagnosed based on morphology, cytochemistry and response to All-trans Retinoic Acid/Arsenic Trioxide (ATRA/ ATO).

Conclusion: APL stands apart from other AMLs because of its clinical features, morphology, and distinct cytogenetic and good response to therapy. Early diagnosis of APL is essential for management of patients for better outcome.

\section{INTRODUCTION}

APL with translocations $t(15 ; 17) \quad(q 22 ; q 12)$ is a leukaemia predominated by abnormal promyelocytes [1]. It differs from other $\mathrm{AML}$ in that; the leukemic cells are not in the blast stage but are atypical promyelocytes [2]. Many of the patients present with bleeding manifestations. This leukaemia is characterised by random karyotype of $t(15 ; 17)$ (q22; q12) with the molecular characteristic of $\mathrm{PML} /$ Retinoic acid receptor $\alpha(\mathrm{RAR} \alpha)$ fusion transcript in the majority of cases [3]. It is one of the rare examples of leukaemia for which an effective treatment has been established by understanding its molecular genetic abnormality. The therapeutic mechanism of AllTrans Retinoic Acid (ATRA) as well as Arsenic Trioxide (ATO) is to induce differentiation of the leukemic cells. Both the classic and microgranular variants have characteristic morphology, which should be identified and communicated to clinician immediately. The 'gold standard' for the diagnosis, is identification of PML-RAR $\alpha$ ' by molecular techniques; hence, this has to be done so that appropriate therapy may be initiated [4]. Objective of this study was to know the clinico-pathological profile of all cases diagnosed as APL and correlate with the cytogenetic and molecular studies. Molecular and cytogenetic analysis in these cases helps in early and accurate diagnosis, identifying translocations and ultimately contributes in selecting chemotherapeutic agents which increases prognosis.

\section{MATERIALS AND METHODS}

This was a retrospective cum prospective analytical study done over the period of five years between January 2010 and June 2015. All retrospective cases of APL were retrieved from haematopathology record files. They were referred to the Pathology department from the Medical Oncology and General Medicine departments, Nizam's Institute of Medical Sciences, Hyderabad, Telangana, India. Institute Ethics committee permission and patient's consent was taken for this study. Patients diagnosed as APL on morphology and cytogenetic/ molecular studies, were included. There were few cases diagnosed as APL on morphology, which responded to ATRA or ATO, and were included in the study, even though they lacked cytogenetic studies. The clinical details, treatment and follow-up details were obtained from Medical Oncology and General Medicine records. The morphology of each case, along with coagulation studies and molecular studies were reviewed. Peripheral blood smears, bone marrows aspirate slides and trephine biopsy imprint slides were studied using Giemsa stain.

Cytochemistry using SBB and Periodic Acid Schiff (PAS) stain was done in all cases. The samples were also submitted for coagulation studies. The coagulation studies with PT, aPTT Fibrinogen and D Dimer levels were performed in all cases. Analysis was performed on fully automated STA Compact Coagulation System of Diagnostica Stago, with PT reagent (Neoplastine CL plus 10), aPTT reagent (STA-C.K. Prest 5), Fibrinogen (STA-FIB 2), D-Dimer (STA-Liatest $\mathrm{D}$-DI) with appropriate control according to the manufacturer protocol $[5,6]$.

Bone marrow samples were studied by flow cytometry using Beckman Coulter, Cytomics FC 500 four colour flowcytometer 
with standard Lyse wash technique. Antibodies used were labeled with Fluorescein Isothiocynate (FITC), Phycoerythrin(PE), Energy Coupled Dye (ECD) and phycoerythrin-cyanine-5 conjugate (PC-5). Antibody panel used was CD13, CD33, CyMPO, CD117, HLADR, CD34, CD14 and CD19. Sensitivity of the antibody panel ranges from 69\% - 99\% and specificity ranges from 67\%-100\%. Antibody was considered positive if more than $20 \%$ of cells gated were positive. Sample was processed within 24 hours of collection. Gating strategy used was CD45 Vs SSC [7-9].

Sample of all bone marrow collected from new and follow-up cases for karyotyping were subjected to culture and harvesting procedure [10]. Subsequently, slide preparation and G-banding was performed prior to karyotyping. Metaphases were counted and analysed under a microscope (Zeiss AXIO Scope, Germany) equipped with Ikaros software (Metasystem). A total of 20 or all available metaphases were counted and analysed especially for translocation related to APL or secondary chromosomal abnormalities. Additional bone marrow harvested smears or bone marrow aspiration smears or bone marrow imprint smears were submitted for FISH studies with Vysis LSI PML/RARA Dual Colour, Dual Fusion Translocation Probe. Following hybridization, the slides were washed SCC/0.3\% NP40 at appropriate temperature, counterstained with DAPI II (Abbott Molecularlnc.) and coverslip was applied. The slide was analysed under a fluorescent microscope (Zeiss AXIOScope, Germany) equipped with Isis software (Metasystem). A total of 200 nonoverlapping nuclei, which were clearly identified and contained unequivocal signals, were counted. A case was considered to be positive for PML-RARA translocation when the 2 fusion and 1 orange and 1 green signals were identified and was considered negative when 2 red and 2 green signals were identified [11,12]. Signals were checked on metaphase cells wherever available. Samples were outsourced for Real Time Quantitative-Polymerase Chain Reaction (RQ-PCR) due to lack of facilities in the centre. $R Q$ $\mathrm{PCR}$ was done for PML-RAR $\alpha$ fusion transcripts and results were compiled [13].

\section{RESULTS}

During the study period from January 2010 to June 2015, 133 cases of APL were diagnosed initially on basis of morphology, of which 125 cases were included in the study. The other cases were excluded, as immunophenotyping and molecular characterisation were not in favor of APL. Of the 125 cases, 73 cases were males and 52 cases were females (M: $F$ ratio 1.4:1). The ages ranged from 3-60 years, with a median age of 35 years. There were no cases in the seventh decade and very few cases in the $1^{\text {st }}$ decade.

In the present study, the commonest presentation was fever in 100 patients (80\%). There were 37 (29.6\%) patients presenting with signs and symptoms of anaemia like generalised weakness, fatigue, giddiness. Gastrointestinal symptoms were seen in 11 patients (8.8\%). One patient presented with lower limb pain and deep vein thrombosis, possibly a consequence of disseminated intravascular coagulation. One unusual feature in the present study is 4 patients (3.2\%), had gum hypertrophy, which is most commonly seen in acute monocytic leukaemia. There were not many significant findings on physical examination, apart from pallor and petechiae. There was only one case each with hepatomegaly and splenomegaly. Three patients $(2.4 \%)$ were found to have cervical lymphadenopathy. On evaluation one patient was found to have neurocysticercosis, which was probably co-incidental. There was one case of APL, diagnosed in a treated case of papillary carcinoma thyroid, 9 years after the first malignancy. There was another case of APL, diagnosed in a treated case of testicular malignancy, 5 years after the first malignancy. In both the cases, primary cancer therapy details were not available.
In this study, when laboratory parameters were considered- majority of patients, 94 (75.2\%), presented with moderate anaemia and $18(14.4 \%)$ patients had severe anaemia. Only 13 patients had normal values of haemoglobin. Majority of patients, 56 (44.8\%) patients presented with leucopenia, while 44 (35.2\%) patients presented with leukocytosis and 25 (20\%) patients had normal leucocyte count [Table/Fig-1a,b]. Majority of the hypergranular variant of APL presented with leucopenia or normal counts; however 6 cases presented with leukocytosis [Table/Fig-2a,b]. Majority of the microgranular variant of APL presented with leucocytosis while 2 cases showing leucopenia [Table/Fig-2c,d]. Most of the patients, 59 patients (47.2\%) presented with severe thrombocytopenia, 56 (44.8\%) patients had moderate thrombocytopenia, 8 (6.4\%) patients had mild thrombocytopenia and only 2 had normal platelet counts. Morphological distribution of the case tabulated in [Table/Fig-3].
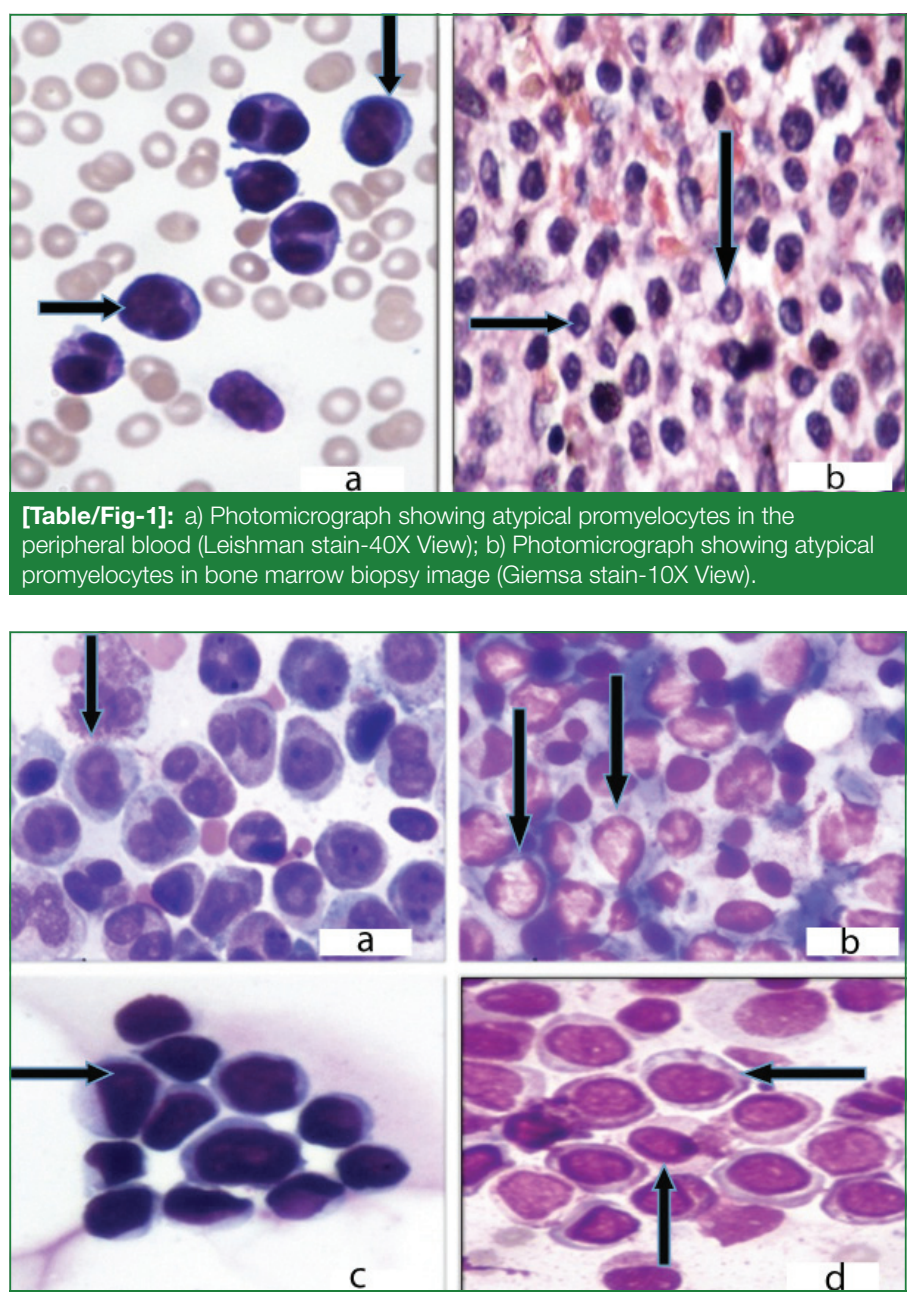

[Table/Fig-2]: a) \& b) Bone marrow aspirate smear demonstrates presence hype granular promyelocytes containing multiple coarse azurophilic granules in the cytoplasm obscuring the nuclear details-Hypergranular APML (Giemsa Stain- 40X View); c) \& d) Bone marrow aspirate smear shows presence of large leukemic cells with abundant cytoplasm very fine dust like azurophilic granules, giving it very finely granular appearance. The blasts have a high Nuclear Cytoplasmic ratio, opened up chromatin and scant amount of cytoplasm and 2-4 prominent nucleoli-Microgranular APML (Giemsa Stain- 40X View).

\begin{tabular}{|l|c|c|}
\hline Type & Number of cases & Percentage \\
\hline Hypergranular/Classic type & 80 & $64 \%$ \\
\hline Microgranular type & 39 & $31.2 \%$ \\
\hline Atypical & 06 & $4.8 \%$ \\
\hline Total & 125 & $100 \%$ \\
\hline [Table/Fig-3]: Morphological distribution of cases.
\end{tabular}

The atypical features included 2 cases showing features of APL with complete maturation upto neutrophils and no evidence of maturation arrest simulating AML M2, but proven later to be APL. There was 1 case in which the cells looked like myelocytes and 
not promyelocytes. There were 3 cases where the morphology was more in favour of monocytic leukaemia but confirmed as APL with molecular evidence. There were no cases of hyperbasophilic variant in the study.

Cytochemistry with SBB and PAS were done in all the cases. SBB showed strong positivity in 100\% of cases, while PAS showed faint granular positivity in 39 (31.2\%) cases while remaining cases were negative [Table/Fig-4a,b].

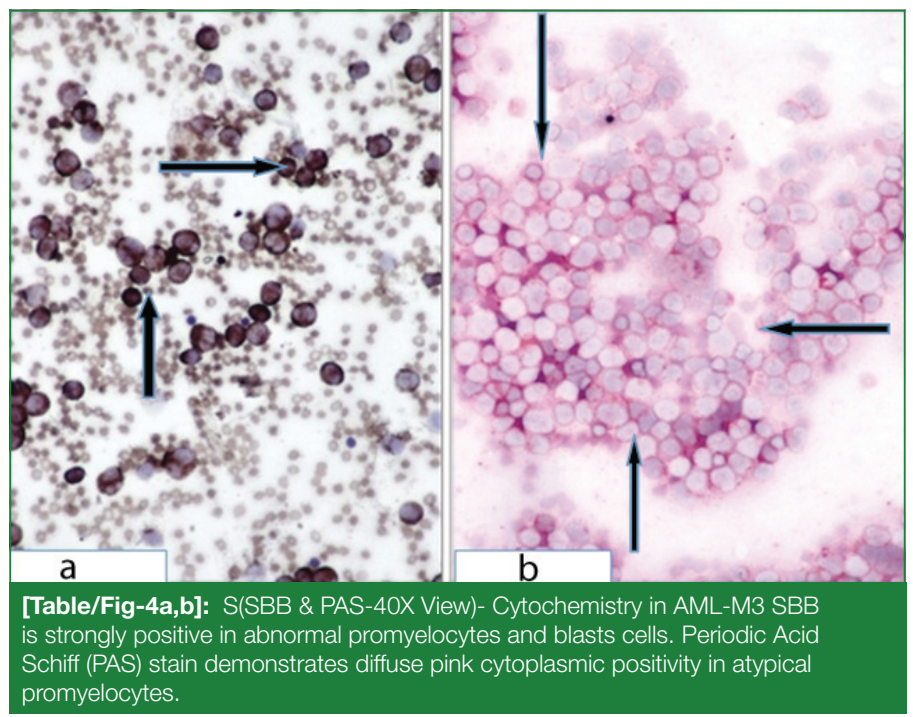

Coagulation profile was done in all cases and the values are depicted in the [Table/Fig-5].

Due to financial constraints faced by the patients, immunophenotyping was done only in 25 cases; the results are represented in [Table/Fig-6].

\begin{tabular}{|l|c|c|c|}
\hline & Increased & Normal & Decreased \\
\hline PT & 91 & 23 & 11 \\
\hline APTT & 72 & 41 & 12 \\
\hline Fibrinogen & 13 & 73 & 39 \\
\hline D Dimer & 119 & 6 & -- \\
\hline \multicolumn{2}{l}{} \\
Trefle
\end{tabular}

\begin{tabular}{|l|c|c|}
\hline Marker & Positive & Negative \\
\hline Cy Mpo & $25 / 25(100 \%)$ & - \\
\hline Cd 13 & $25 / 25(100 \%)$ & - \\
\hline Cd 33 & $25 / 25(100 \%)$ & - \\
\hline Cd 117 & $25 / 25(100 \%)$ & - \\
\hline Hla Dr & $7 / 25(28 \%)$ & $18 / 25(72 \%)$ \\
\hline Cd 34 & $4 / 25(16 \%)$ & $21 / 25(84 \%)$ \\
\hline
\end{tabular}

Due to the necessity for speedy results, only 13 samples were submitted for conventional cytogenetics [8]. It failed in 4 cases and 2 show poor banding while two cases show balanced reciprocal translocation t (15; 17). Translocation results, done by different techniques, were available in 90 (72\%) cases. Out of 90 cases, 24 cases (26.6\%) were analysed by FISH technique at our Institute, in which 22 cases were positive for PML RARA and 2 cases were negative. In two cases, FISH showed atypical signals as single fusion or 3 red and 2 green signals [11]. [Table/Fig-7] depicts the FISH results of a case of APL.

By PCR technique, 56 cases (62.2\%) were analysed; out of which 54 cases were positive for PML-RARA and 2 cases were negative. In 10 out of 90 cases (11.1\%), both techniques were applied out of which 4 cases showed translocation by both techniques. In 1 case, PML-RAR $\alpha$ was detected by FISH technique and not detected by PCR technique, while in 5 cases translocation was detected by PCR technique and not detected by FISH technique.

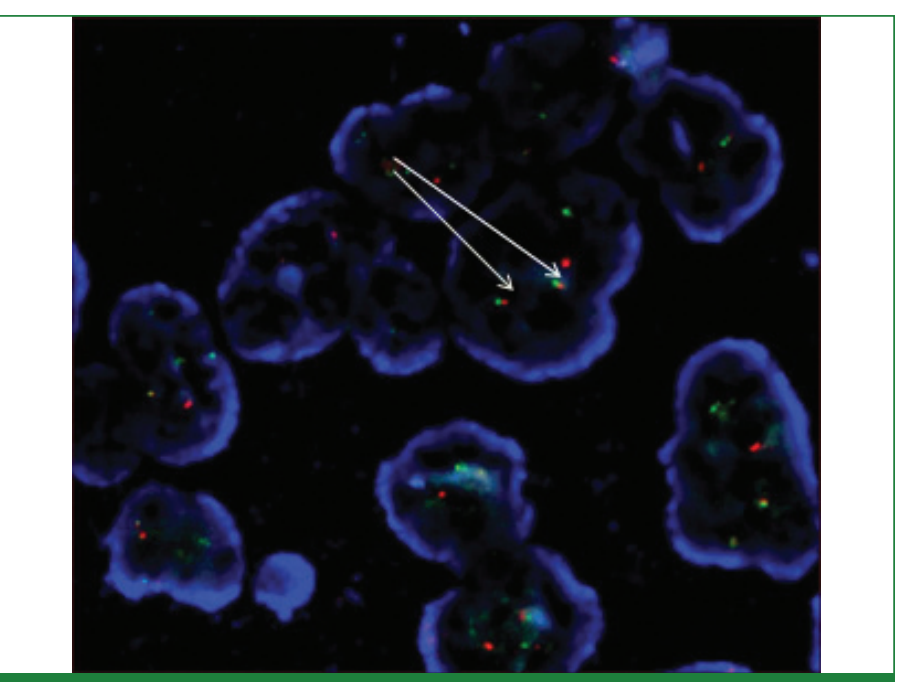

[Table/Fig-7]: FISH study showing PML-RARA dual color translocation probe (Nysis)-2 fusion (PML/RARA) signal.

\begin{tabular}{|l|c|c|}
\hline & Present study & Dutta P et al., [14] \\
\hline Number of cases & 125 & 35 \\
\hline Peak age incidence & $31-40$, Median age=35 Years & $11-20$ \\
\hline Fever & $80 \%$ & $68 \%$ \\
\hline Bleeding tendencies & $55.2 \%$ & $83 \%$ \\
\hline Hypergranuar variant & $64 \%$ & $100 \%$ \\
\hline Microgranular variant & $31.2 \%$ & -- \\
\hline SBB & $100 \%$ & $100 \%$ \\
\hline PAS & $31.2 \%$ & Not done \\
\hline NSE & Not done & $61 \%$ \\
\hline PML RARA Positivity & $95.5 \%$ & $94.2 \%$ \\
\hline
\end{tabular}

[Table/Fig-8]: Comparison between the present study and that by Dutta P et al., [14].

\begin{tabular}{|l|c|c|}
\hline Parameter & Present study & Lee HJ et al., [15] \\
\hline Number of patients & 125 & 14 \\
\hline Median age & 35 years & 49 years \\
\hline Fibrinogen decreased & $39 / 125$ & $19 / 19$ \\
\hline D Dimer elevated in & $119 / 125$ & $19 / 19$ \\
\hline
\end{tabular}

\section{DISCUSSION}

APL stands apart from other AMLs because of its characteristic morphology, bleeding tendency, chromosomal translocation and good response to therapy, if it is diagnosed in time. This study includes 125 cases of APL. During the study period, APLs accounted for $20 \%$ of all AMLs, which is much higher than the reported rate of $5-6 \%$. This is probably due to a referral bias, as our Institute is a tertiary care center and the more serious patients are referred here. The disease seems to occur in crops, with many patients presenting together and then long gaps in between. We have no explanation for this. In the present study, there is male preponderance with peak incidence in fourth decade and the incidence below the age of 10 years and after 60 years is low.

Majority of patients in this study came with fever as presenting symptom and on evaluation with bone marrow studies were diagnosed as APL; this is different from previous study conducted by Dutta $P$ et al., in which majority of patients presented with bleeding manifestations [Table/Fig-8] shows the comparison between the present study and study done by Dutta P et al., [14]. The cause of fever is thought to be due to infections arising in the background of leucopenia or abnormal promyelocytes and reduced normal neutrophils. Four of the patients were absolutely asymptomatic, and on evaluation for trivial reasons, were found to have APL. One patient presented as stroke in young and on peripheral smear evaluation found to have abnormal promyelocytes, while 
one more patient came with lower limb deep vein thrombosis, for which he was evaluated and found to have APL. One patient came with history of prolonged bleeding after tooth extraction and was diagnosed as APL. There was a peculiar case of a patient who developed lower limb cellulitis after an insect bite and interestingly on routine investigations found to have APL. After fever, most of the patients presented with bleeding manifestations in the form of bleeding gums, menorrhagia, melaena, nose bleeds and petechiae all over the body. There were considerable number of patients who came with signs and symptoms of anaemia.

Two patients had previous malignancies- one had papillary carcinoma thyroid while other has carcinoma testis. As a detailed therapeutic history was not available, these were not labeled as therapy-related AMLs. Organomegaly like splenomegaly and hepatomegaly were infrequent as in other studies. An unusual feature in the present study was gum hypertrophy seen in four patients. This is usually noted in the monocytic leukaemias, but has been reported in the Indian study by Dutta $P$ et al., [14]. On peripheral blood evaluation, most of the patients had anaemia and thrombocytopenia. Total white blood cell count varied according to the morphological variant of APL. Twenty five patients had normal range of total WBC count.

Morphologically, the present study had a peculiar feature as microgranular variant of APL constituted $31.2 \%$ which is high when compared with various previous studies like Dutta et al., where the study was done on 35 patients and all were diagnosed as typical/ hypergranular variant of APL [14]. On cytochemistry, SBB is positive in $100 \%$ of cases which is in accordance with review of literature.

Regarding the DIC profile, majority of cases showed elevated PT, APTT levels, but most of the cases show normal levels of fibrinogen which is against the finding of hypofibrinogenemia, generally noted in acute promyelocytic leukaemia. In the study by Lee $\mathrm{HJ}$ et al., 100\% cases showed hypofibrinogenemia. D- Dimer levels are elevated in 99\% of the cases. [Table/Fig-9] shows the comparison between the present study and study done by Lee $\mathrm{HJ}$ et al., [15].

In the present study immunophenotyping, Cy MPO, CD 117, CD 13, CD 33 were positive in 100\% cases, which is according to literature, while HLA-DR, CD 34 which are usually negative in APL were positive in a few cases. In a study conducted by Ansari R et al., at Tata Memorial Hospital, CD 34 and HLADR were absent in all cases of APL [16]. In the present study cytogenetic translocation was detected in significant number, $95.5 \%$, of cases. The major limitation of present study is very few of the cases have conventional karyotyping details as they are time-consuming and treatment should be initiated as early as possible to save the patients. Hence, most of the cases were diagnosed by molecular techniques alone without opting for conventional cytogenetics. Majority were diagnosed by PCR technique rather than FISH. In FISH, majority were done on bone marrow aspirate while in five cases it was done on peripheral blood harvested material because those cases had high WBC count and in four cases it was done on trephine imprint as APL was not suspected prior to the procedure. The cases which were negative for PML-RAR $\alpha$, were also considered as APL as they went into remission with induction chemotherapy either by ATRA or ATO.

Out of 78 patients who received either ATRA or ATO and on regular follow-up, $62 \%$ are doing well. However, 10 patients relapsed within time range of 10-36 months and were started with re-induction chemotherapy. In the relapsed patients, three underwent bone marrow transplant and are presently doing well. The eight deaths that occurred in the first week of admission were due to intracranial bleeds. Differentiation syndrome was seen in 45 cases and they were treated with dexamethasone.

\section{TREATMENT AND FOLLOW-UP}

After the diagnosis, 37 patients were treated with Arsenic Trioxide (ATO), 39 patients were treated with ATRA (All Trans Retinoic Acid) and two patients were treated with both drugs. Due to financial constraints, another 39 patients did not opt for therapy and were lost to follow-up. These patients Left Against Medical Advice (LAMA) once the prognosis and monetary burden was explained. There were eight deaths within 3-4 days of admission. Of the 125 patients, $62.4 \%$, (78 of 125) patients are on regular follow-up, following completion of induction, consolidation and maintenance phases of treatment. These patients are now on close hematologic follow-up with regular peripheral blood examinations and molecular studies.

\section{LIMITATION}

Due to financial constraints Immunophenotyping was done only in few cases and few patients did not opt for therapy and left against medical advice; eight patients died within few days of admission.

\section{CONCLUSION}

APL are a medical emergency; the pathologist on duty should be able to suspect the diagnosis so that further investigations and appropriate treatment are given in time to save the patient. Early diagnosis and appropriate therapy has changed the disease from highly fatal to highly curable condition.

\section{REFERENCES}

[1] Arber DA, Brunning RD, Le Beau MM, Falini B, Vardiman JW, Portwit A, et al Acute Myeloid Leukaemia with recurrent genetic abnormalities in: Swerdlow $\mathrm{SH}$, Campo E, Harris NL et al (eds). WHO Classification of Tumours of Haematopoietic and Lymphoid organs. $4^{\text {th }}$ ed. Lyon, France: IARC press 2008 , p 112-14.

[2] Akhtar K, Ahmad S, Sherwani RK. Acute promyelocytic leukemia, hypogranular variant: A rare presentation. Clin Pract. 2011;1(1):e11. Published 2011 Apr 12. doi:10.4081/cp.2011.e11

[3] Zhang R, Kim Y, Wang X, Li Y, Pang H, Lee J, et al. Coexistence of $t(15 ; 17)$ and $t(15 ; 16 ; 17)$ detected by fluorescence in situ hybridization in a patient with acute promyelocytic leukemia: A case report and literature review. Oncology Letters. 2014;8:1001-08.

[4] Nowak D, Stewart D, Koeffler HP. Differentiation therapy of leukemia: 3 decades of development. Blood. 2009;113(16):3655-65. doi:10.1182/blood-2009-01198911.

[5] Martin S, Tallman MS, Kwaan HC. Reassessing the hemostatic disorder associated with acute promyelocytic leukemia. Blood. 1992;79:543-53.

[6] Sterrenberg L, Haak HL, Brommer EJP, Nieuwenhuizen W. Evidence of fibrinogen breakdown by leukocyte enzymes in a patient with acute promyelocytic leukemia. Haemostasis. 1985;15:126-31.

[7] Edwards RH, Wasik MA, Finan J, Rodriguez R, Moore J, Kamoun M, et al. Evidence of early haematopoietic progenitor cell involvement in acute promyelocytic leukemia. Am J Clin Pathol. 1999;112:819-27.

[8] Orfao A, Chillon MC, Bortoluci AM, López-Berges MC, García-Sanz R, Gonzalez $M$, et al. The flow cytometric pattern of CD 34, CD 15 and CD 13 expression in acute myeloblastic leukemia is highly characteristic of the presence of PML RAR alpha gene rearrangements. Haematologica 1999;84:405-12.

[9] Rizzatti EG, Garcia AB, Pothan H, Silva DE, Martins SL, Falcão RP. Expression of CD117 and CD 11b in bone-marrow can differentiate acute promyelocytic leukemia from recovering myeloid proliferations. Am J ClinPathol. 2002;118:31-37

[10] Misawa S, Lee E, Schiffer CA, Liu Z, Testa J R. Association of translocation $(15 ; 17)$ with malignant proliferation of promyelocytes in acute leukemia and chronic myelogenous leukemia in blast crisis. Blood. 1986;67:270-74.

[11] Kakizuka A, MillerJr WH, Umesono K, Warrell Jr. RP, Frankel SR, Murty VVVS, et al. Chromosomal translocation $\mathrm{t}(15 ; 17)$ in human acute promyelocytic leukemia fuses RAR $\alpha$ with a novel putative transcription factor, PML. Cell. 1991;23:663-74.

[12] Weis K, Rambaud S, Lavau C, Jansen J, Carvalho T, Lamond A, et al. Retinoic acid regulates aberrant nuclear localization of PML-RAR $\alpha$ in acute promyelocytic leukemia cells. Cell. 1994;28:345-56.

[13] Grignani F, Fagioli M, Alcalay M, Longo L, Pandolfi PP, Do E, et al. Acute promyelocytic leukemia. From genetics to treatment. Blood. 1994;83:10-25.

[14] Dutta P, Sazawal S, Kumar R, Saxena R. Does acute promyelocytic leukemia in Indian patients have biology different from west? IJPM. 2008;51:437-39. 
[15] Lee HJ, Park HJ, Kim HW, Park SG. Comparision of laboratory characteristics between acute promyelocytic leukemia and other subtypes of acute myeloid leukemia with disseminated intravascular coagulation. Blood. 2013;48:250-53.
[16] Ansari R, Badrinath $Y$, Chougule AB, Dhond SR, et al. Haematologic and immunophenotypic profile of acute myeloid leukemia: an experience of Tata Memorial Hospital. Indian Journal of Cancer. 2003;40:71-76.

PARTICULARS OF CONTRIBUTORS:

1. Postgraduate, Department of Pathology, Nizam's Institute of Medical Sciences, Hyderabad, Telangana, India.

2. Assistant Professor, Department of Pathology, Nizam's Institute of Medical Sciences, Hyderabad, Telangana, India.

3. Associate Professor, Department of Pathology, Nizam's Institute of Medical Sciences, Hyderabad, Telangana, India

4. Associate Professor, Department of Pathology, Nizam's Institute of Medical Sciences, Hyderabad, Telangana, India.

5. Professor, Department of Pathology, Nizam's Institute of Medical Sciences, Hyderabad, Telangana, India.

6. Professor, Department of Hematology, Nizam's Institute of Medical Sciences, Hyderabad, Telangana, India.

7. Additional Professor, Department of Medical Oncology, Nizam's Institute of Medical Sciences, Hyderabad, Telangana, India.

8. Professor and Head, Department of Pathology, Nizam's Institute of Medical Sciences, Hyderabad, Telangana, India.

\section{NAME, ADDRESS, E-MAIL ID OF THE CORRESPONDING AUTHOR:}

Dr. T Roshni Paul,

Professor and Head, Department of Pathology, $5^{\text {th }}$ Floor, Millennium Block, NIMS Hospital,

Punjagutta, Hyderabad, Telangana-500082, India.

E-mail: pramodkumarpamunims@gmail.com; troshnip@yahoo.co.in

FINANCIAL OR OTHER COMPETING INTERESTS: None.

Date of Submission: Jul 20, 2019

Date of Peer Review: Jul 31, 2019

Date of Acceptance: Aug 17, 2019

Date of Publishing: Oct 01, 2019 